\title{
Structural and functional assessment of macula to diagnose glaucoma
}

HL Rao1,2, RSM Hussain¹, M Januwada1, LN Pillutla1, VU Begum¹ , A Chaitanya ${ }^{1}$, S Senthil ${ }^{1}$ and CS Garudadri ${ }^{1}$

\begin{abstract}
Purpose To compare the diagnostic abilities of structural (ganglion cell-inner plexiform layer (GCIPL) thickness measured using spectral domain optical coherence tomography (SDOCT)) and functional (visual sensitivities measured using standard automated perimetry (SAP) and microperimetry (MP)) assessments of macula in glaucoma.

Methods In a prospective study, 46 control eyes (28 subjects) and 61 glaucoma eyes (46 patients) underwent visual sensitivity estimation at macula (central $10^{\circ}$ ) by SAP and MP, and GCIPL thickness measurement at macula by SDOCT. Glaucoma was diagnosed by experts based on the optic disc and retinal nerve fiber layer changes. Area under the receiver-operating characteristic (AUC) curves and sensitivities at $95 \%$ specificity were used to assess the diagnostic ability of visual sensitivity and GCIPL measurements at various macular sectors.
\end{abstract}

Results AUCs of GCIPL parameters ranged between 0.58 and 0.79 . AUCs of SAP and MP sensitivities ranged between 0.59 and 0.71 , and 0.59 and 0.72 , respectively. There were no statistically significant differences between the AUCs of corresponding sector measurements ( $\boldsymbol{P}>\mathbf{0 . 1 0}$ for all comparisons). Sensitivities at $95 \%$ specificities ranged from $31-59 \%$ for GCIPL parameters, $16-34 \%$ for SAP, and $8-38 \%$ for MP parameters. Sensitivities were significantly better with GCIPL compared with SAP and MP parameters in diagnosing glaucoma. Inferotemporal, inferior, and superotemporal sector measurements of GCIPL and visual sensitivity showed the best abilities to diagnose glaucoma.

Conclusions Comparing the diagnostic abilities of structural and functional tests at macula in glaucoma, GCIPL thickness measurements with SDOCT performed better than the visual sensitivity measurements by SAP and MP.

Eye (2017) 31, 593-600; doi:10.1038/eye.2016.277; published online 9 December 2016

\section{Introduction}

Glaucoma is traditionally defined as a chronic, progressive optic neuropathy with characteristic optic nerve head $(\mathrm{ONH})$ and retinal nerve fiber layer (RNFL) changes, with or without correlating visual field (VF) defects. However, with the advent of spectral domain optical coherence tomography (SDOCT), another region that has been shown to demonstrate structural changes in glaucoma is the macula. Imaging the inner retinal layers at macula has evolved as a useful modality to diagnose glaucoma. ${ }^{1-9}$ Standard automated perimetry (SAP) has been the preferred method to evaluate the defects in the central $24^{\circ}$ or $30^{\circ} \mathrm{VF}$ in glaucoma. The commonly used program to evaluate the central $24^{\circ}$ of VF tests the visual sensitivities at 54 points that are $6^{\circ}$ apart. However, the macular region (central $5^{\circ}-10^{\circ}$ ) is tested using only 8-16 points in this program which are $6^{\circ}$ apart. Also, the locations of the ganglion cells stimulated by the central 16 points of the $24^{\circ}$ program are farther from the fovea because the ganglion cells in the fovea are displaced. ${ }^{10,11}$ The sampling density of central $24^{\circ}$ program to estimate visual sensitivity at macula is also inadequate. A study by Schiefer et $a l^{12}$ has shown better detection of VF defects in eyes with suspected glaucoma when closely spaced test points were used. In addition to the $24^{\circ}$ program, SAP also has a program to examine the central $10^{\circ}$ of the VF with higher resolution. This program evaluates the central $10^{\circ}$ of VF using 68 test points that are $2^{\circ}$ apart. Traynis et al ${ }^{13}$ have reported field defects on $10^{\circ}$ program in eyes with no defects on $24^{\circ} \mathrm{VF}$ program.

In addition to the SAP, another relatively newer method of estimating visual sensitivities at retinal points is the microperimetry (MP).
${ }^{1}$ VST Glaucoma Center, LV Prasad Eye Institute, Hyderabad, India

${ }^{2}$ Narayana Nethralaya, Rajajinagar, Bangalore, India

Correspondence: HL Rao, VST Glaucoma Center, LV Prasad Eye Institute, Kallam Anji Reddy Campus, Banjara Hills, Hyderabad 500034, India Tel: +91 8066121318 ; Fax: +91 4023548271. E-mail: harshalaxmanarao@ gmail.com

Received: 18 August 2015 Accepted in revised form: 24 October 2016 Published online: 9 December 2016 
MP, which is also known as fundus perimetry, assesses retinal sensitivity while directly examining the ocular fundus. MP results are independent of eye movements and exactly related to the stimulated area. ${ }^{14}$ Therefore, the VF sensitivities measured by MP is supposed to have better spatial localization.

Although there are multiple studies evaluating the diagnostic ability of inner retinal layers at the macula in glaucoma (as referenced earlier), there are no studies comparing the diagnostic abilities of inner retinal layers with that of the functional evaluation of the macula with either the SAP or MP. The purpose of our study was to compare the abilities of structural (inner retinal layer thickness measured using SDOCT) and functional (visual sensitivities measured using SAP and MP) assessments of macula in diagnosing glaucoma.

\section{Materials and methods}

This was a prospective, cross-sectional study conducted at a tertiary eye care center between January 2015 and April 2015 to evaluate the structural and functional changes at macula in glaucoma. Written informed consent was obtained from all participants and the Ethics Committee of LV Prasad Eye Institute approved the study methodology. All methods adhered to the tenets of the Declaration of Helsinki for research involving human subjects.

Inclusion criteria were age $\geq 18$ years, best-corrected visual acuity of 20/40 or better and refractive error within $\pm 5 \mathrm{D}$ sphere and $\pm 3 \mathrm{D}$ cylinder. Exclusion criteria were the presence of any media opacities that affected SDOCT scans and SAP and MP results, and any retinal (including macular) or neurological disease other than glaucoma, that could confound the evaluations. All participants underwent a comprehensive ocular examination, which included a detailed medical history, best-corrected visual acuity measurement, slit-lamp biomicroscopy, Goldmann applanation tonometry, gonioscopy, dilated fundus examination, central VF examination with SAP and MP, and SDOCT imaging with Cirrus high-definition OCT (HD-OCT; Carl Zeiss Meditec Inc., Dublin, CA, USA).

SDOCT examination was performed with Cirrus HDOCT (software version 6.0). Macular Cube $200 \times 200$ protocol was used to image the macula. This protocol has been explained in detail previously. ${ }^{15,16}$ Ganglion cell analysis (GCA) is a software that measures the ganglion cell-inner plexiform layer (GCIPL) thickness within a $14.13 \mathrm{~mm}^{2}$ elliptical annulus centered on the fovea with an inner vertical radius of $0.5 \mathrm{~mm}$ and outer vertical radius of $2 \mathrm{~mm}$, stretched horizontally by $20 \%$. The thickness parameters derived from GCA are the average GCIPL thickness across the entire elliptical annulus and the thickness at six $60^{\circ}$ sectors of the elliptical annulus.
Scans with signal strength $<6$ or with segmentation errors were excluded from analysis.

SAP examination of the macula was performed using a Humphrey Field analyzer, model 750i (Zeiss Humphrey Systems, Dublin, CA, USA), with the Swedish interactive threshold algorithm standard 10-2 program. VFs were considered reliable if the fixation losses were $\leq 20 \%$ and false-positive and -negative response rates were $\leq 15 \%$.

MP examination of the macula was performed using the Macular Integrity Assessment (CenterVue, Padova, Italy). The protocol used was a 10-2 grid that tests the retinal sensitivity in the central $10^{\circ}$ of the retina using 68 points, similar to the 10-2 program of SAP. The stimulus size was equal to the Goldmann size III stimulus of SAP. Background luminance was set at 4 apostilb, with a maximum luminance of 1000 apostilb and a stimulus dynamic range of $36 \mathrm{~dB}$. Visual sensitivities were estimated using a 4-2 threshold strategy. MP results were considered reliable if the reliability index was $>70 \%$ (percentage of control points projected on the optic nerve that were not seen by the subject) and the fixation was stable ( $>75 \%$ of the fixation points were located within a $2^{\circ}$ diameter circle centered in the gravitational center of all fixation points).

To evaluate the GCIPL thickness and visual sensitivity at exactly similar locations at macula, we used the structure-function correlation map described previously using the GCIPL sectors of Cirrus HD-OCT and the 10-2 program of SAP and MP. ${ }^{17}$ This map also considered the displacement of the RGCs at the macula by using equations derived from the histological analysis to approximate the location of the RGCs with each SAP test point. ${ }^{10}$ Visual sensitivities at all 68 points of SAP and MP were first converted from the $\mathrm{dB}$ scale to a linear scale (reciprocal of Lambert scale) using the following formula.

$\frac{1}{\text { Lambert }}=(10)^{0.1 * \mathrm{~d} B}$

The values from all test points within the VF sectors corresponding to the anatomic sectors described above were then averaged. The average visual sensitivity per sector was converted back to the $\mathrm{dB}$ scale for the analysis. This procedure has been explained in detail previously. ${ }^{18}$ The sectors of SAP were flipped horizontally and vertically to correlate with the sectors of HD-OCT and MP.

Glaucoma group consisted of a consecutive sample of eligible glaucoma patients attending the hospital during the study period. Glaucoma was diagnosed in all these eyes by the treating glaucoma specialist based on the characteristic optic discs findings of neuroretinal rim thinning, notching, or localized or diffuse RNFL defects. We also included a control group that consisted of subjects attending the hospital for a routine eye check for 
refractive error or were spouses and friends of the recruited patients. Control subjects had no family history of glaucoma, no history of raise in IOP in the past, normal anterior and posterior segments as assessed by experts on clinical examination, and IOP $\leq 21 \mathrm{~mm} \mathrm{Hg}$. Neither VF nor SDOCT results were used in the definition of glaucoma. Both the VF examinations (SAP and MP) and the SDOCT examination were carried out on the same day in all the subjects.

\section{Statistical analysis}

Descriptive statistics included mean and standard deviation for normally distributed variables and median and interquartile range (IQR) for non-normally distributed variables. Receiver-operating characteristic (ROC) curves and sensitivity at a fixed specificity of $95 \%$ were used to describe the ability of the different structural and functional parameters to discriminate glaucomatous eyes from control eyes. To obtain confidence intervals for area under the ROC curves (AUCs) and sensitivities, a bootstrap resampling procedure was used ( $n=1000$ resamples). As measurements from both eyes of the same subject are likely to be correlated, the standard statistical methods for parameter estimation lead to underestimation of standard errors and to confidence intervals that are too narrow. ${ }^{19}$ Therefore, the cluster of data for the study subject was considered as the unit of resampling and bias-corrected standard error was calculated during all estimations. This procedure has been used in literature to adjust for the presence of multiple correlated measurements from the same unit. ${ }^{20,21} Z$-test was used to compare the AUCs and tests of proportions were used to compare the sensitivities at $95 \%$ specificities of structural and functional parameters. $^{22,23}$

Statistical analyses were performed using the commercial software (Stata ver. 12.0; StataCorp, College Station, TX, USA). A $P$-value $<0.05$ was considered statically significant.

\section{Results}

One hundred and twenty-seven eyes of 80 participants (53 eyes of 31 control subjects and 74 eyes of 49 glaucoma patients) underwent GCIPL imaging with HD-OCT and macular visual sensitivity assessment with SAP and MP. After excluding the poor quality HD-OCT, SAP, and MP tests (shown in the Venn diagram; Figure 1), final analysis included 108 eyes of 72 participants. Table 1 shows the age, GCIPL thickness, SAP, and MP sensitivities of the glaucoma and the control groups. Control subjects were significantly younger than the glaucoma patients. AUCs and sensitivities at fixed specificity, therefore, were

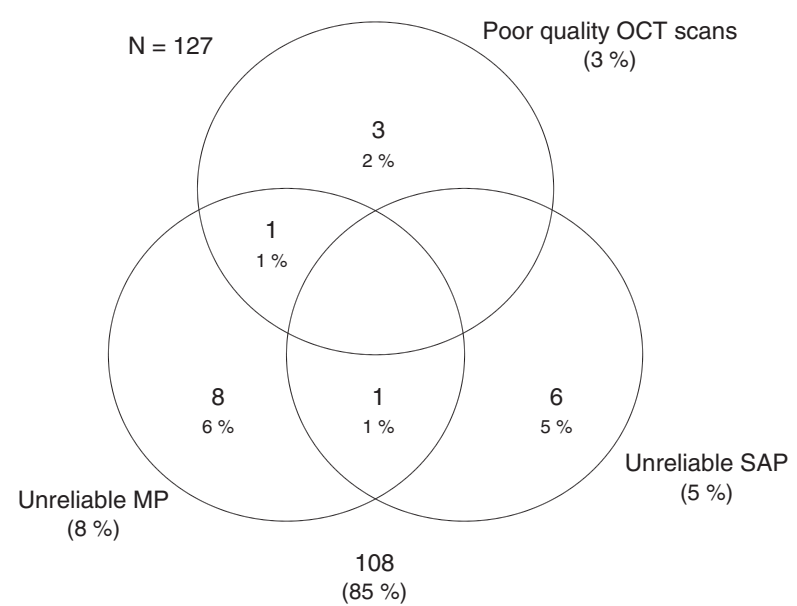

Figure 1 Venn diagram showing the number of eyes excluded from the analysis because of poor test results.

adjusted for the differences in the age between the control and the glaucoma groups using covariate adjustment, as proposed by Pepe. ${ }^{24}$ On evaluating the 24-2 SAP results and the mean deviation (MD) of the 24-2 SAP of the 61 eyes with the glaucoma, 9 had preperimetric, 15 early (MD of better than $-6 \mathrm{~dB}$ ), 16 moderate (MD between -6 and $-12 \mathrm{~dB}$ ), and 21 severe (MD of worse than $-12 \mathrm{~dB}$ ) VF loss.

The AUCs of structural and functional parameters to differentiate glaucoma from control eyes are shown in Table 2. Among the parameters, inferotemporal, inferior, and superotemporal sectors along with the overall average measurements of GCIPL thickness and visual sensitivities showed the best AUCs to diagnose glaucoma. Table 2 also shows the $P$-values associated with the comparisons between the structural and functional parameters. Although the AUCs of most GCIPL parameters were better than that of the functional measurements of SAP and MP, none of the differences were statistically significant.

Sensitivities at $95 \%$ specificity of structural and functional parameters to differentiate glaucoma from control eyes are shown in Table 3. Among the parameters, inferotemporal, inferior, and superotemporal sectors along with the overall average measurements of GCIPL thickness and visual sensitivities showed the best sensitivities to diagnose glaucoma. Table 3 also shows the $P$-values associated with the comparisons between the structural and functional parameters. Sensitivities of GCIPL parameters were significantly better than that of the corresponding visual sensitivity parameters of SAP and MP.

Figure 2 shows the ROC curves of the structural and functional parameters for the superotemporal 
Table 1 Structural and functional characteristics of the participants

\begin{tabular}{|c|c|c|c|}
\hline & Control group (47 eyes of 28 subjects) & Glaucoma group (61 eyes of 44 subjects) & P-value \\
\hline Age (years) ${ }^{a}$ & $48.3 \pm 12.7$ & $54.9 \pm 11.7$ & 0.03 \\
\hline \multicolumn{4}{|c|}{ Humphrey visual field parameters (dB) } \\
\hline Mean deviation & $-2.1(-4.2,-0.5)$ & $-7.6(-18.6,-4.4)$ & $<0.001$ \\
\hline Pattern standard deviation & $1.4(1.2,3.2)$ & $3.7(1.4,9.6)$ & $<0.001$ \\
\hline Superotemporal sensitivity & $30.4(28.0,32.5)$ & $28.5(25.8,30.9)$ & 0.01 \\
\hline Superior sensitivity & $30.6(27.7,32.3)$ & $29.0(26.6,31.5)$ & 0.06 \\
\hline Superonasal sensitivity & $31.0(27.7,32.8)$ & $29.9(27.6,32.1)$ & 0.24 \\
\hline Inferonasal sensitivity & $30.4(28.3,32.3)$ & $29.1(25.6,30.8)$ & 0.07 \\
\hline Inferior sensitivity & $29.6(28.4,31.5)$ & $27.2(18.7,29.8)$ & $<0.001$ \\
\hline Inferotemporal sensitivity & $30.5(28.1,32.2)$ & $27.7(18.3,30.5)$ & $<0.001$ \\
\hline Average sensitivity & $30.3(27.6,32.3)$ & $28.7(24.6,30.5)$ & 0.004 \\
\hline \multicolumn{4}{|l|}{ GCIPL parameters $(\mu m)$} \\
\hline Superotemporal thickness & $77(73,81)$ & $65(52,74)$ & $<0.001$ \\
\hline Superior thickness & $77(72,83)$ & $69(56,78)$ & $<0.001$ \\
\hline Superonasal thickness & $80(76,84)$ & $76(63,83)$ & 0.02 \\
\hline Inferonasal thickness & $79(75,83)$ & $72(58,81)$ & $<0.001$ \\
\hline Inferior thickness & $76(73,83)$ & $63(53,75)$ & $<0.001$ \\
\hline Inferotemporal thickness & $78(73,83)$ & $60(50,72)$ & $<0.001$ \\
\hline Average thickness & $77(74,83)$ & $68(55,78)$ & $<0.001$ \\
\hline \multicolumn{4}{|l|}{ Microperimetry parameters $(d B)$} \\
\hline Superotemporal sensitivity & $26.8(22.9,28.5)$ & $23.4(20.2,25.7)$ & 0.001 \\
\hline Superior sensitivity & $26.0(23.8,28.1)$ & $24.2(22.4,26.4)$ & 0.01 \\
\hline Superonasal sensitivity & $25.5(22.3,28.3)$ & $23.9(21.4,26.7)$ & 0.05 \\
\hline Inferonasal sensitivity & $25.9(21.5,28.1)$ & $23.1(17.7,25.9)$ & 0.002 \\
\hline Inferior sensitivity & $25.0(22.9,27.4)$ & $20.3(0.4,23.5)$ & $<0.001$ \\
\hline Inferotemporal sensitivity & $26.0(22.1,27.9)$ & $20.9(6.2,24.7)$ & $<0.001$ \\
\hline Average sensitivity & $26.4(22.9,28.1)$ & $23.1(20.9,25.6)$ & $<0.001$ \\
\hline
\end{tabular}

Abbreviations: $\mathrm{dB}$, decibel; GCIPL, ganglion cell-inner plexiform layer.

All values are represented as median and interquartile range, unless otherwise specified. ${ }^{a}$ Mean plus/minus SD.

Table 2 Area under the receiver-operating characteristic curves ${ }^{\mathrm{a}}$ of the structural and functional parameters to diagnose glaucoma

\begin{tabular}{lllllll}
\hline Sector & GCIPL thickness & SAP sensitivity & MP sensitivity & P_1 & P_2 & P_3 \\
\hline Superotemporal & $0.76(0.62-0.86)$ & $0.66(0.53-0.78)$ & $0.66(0.53-0.79)$ & 0.21 & 0.19 & 0.99 \\
Superior & $0.68(0.54-0.81)$ & $0.62(0.47-0.73)$ & $0.61(0.46-0.74)$ & 0.44 & 0.38 & 0.93 \\
Superonasal & $0.58(0.43-0.70)$ & $0.59(0.45-0.72)$ & $0.59(0.46-0.72)$ & 0.94 & 0.91 & 0.98 \\
Inferonasal & $0.67(0.54-0.79)$ & $0.63(0.50-0.75)$ & $0.65(0.52-0.75)$ & 0.64 & 0.79 & 0.74 \\
Inferior & $0.77(0.66-0.87)$ & $0.71(0.58-0.82)$ & $0.70(0.56-0.82)$ & 0.50 & 0.35 & 0.80 \\
Inferotemporal & $0.79(0.67-0.89)$ & $0.71(0.58-0.82)$ & $0.72(0.58-0.83)$ & 0.27 & 0.29 & 0.93 \\
Average & $0.74(0.61-0.85)$ & $0.68(0.56-0.79)$ & $0.66(0.53-0.79)$ & 0.40 & 0.29 & 0.81 \\
\hline
\end{tabular}

Abbreviations: GCIPL, ganglion cell-inner plexiform layer; MP, microperimetry; P_1, $P$-value associated with the comparison of the AUCs between GCIPL and SAP parameters; P_2, $P$-value associated with the comparison of the AUCs between GCIPL and MP parameters; P_3, $P$-value associated with the comparison of the AUCs between SAP and MP parameters; SAP, standard automated perimetry. ${ }^{a} 95 \%$ Confidence intervals within parentheses.

(a), inferotemporal (b), and inferior (c) sectors and overall average (d) measurement.

\section{Discussion}

In this study to compare the diagnostic abilities of structural and functional tests at macula in glaucoma, the GCIPL thickness measurements with HD-OCT performed significantly better than the visual sensitivity measurements by SAP and MP.

Studies comparing the structural and functional evaluation of macula in diagnosing glaucoma are sparse. Ozturk et al ${ }^{25}$ compared the macular sensitivities with 10-2 SAP and an older version of MP, and the macular retinal thickness with confocal scanning laser ophthalmoscope (CSLO) in a group of glaucoma patients 
Table 3 Sensitivity at $95 \%$ specificity ${ }^{\mathrm{a}}$ of the structural and functional parameters to diagnose glaucoma

\begin{tabular}{|c|c|c|c|c|c|c|}
\hline Sector & GCIPL thickness & SAP sensitivity & MP sensitivity & P_1 & P_2 & $P \_3$ \\
\hline Superotemporal & $59 \%(41-77)$ & $20 \%(05-49)$ & $21 \%(12-39)$ & $<0.001$ & $<0.001$ & 0.85 \\
\hline Superior & $38 \%(18-58)$ & $21 \%(07-41)$ & $11 \%(03-27)$ & 0.001 & $<0.001$ & 0.01 \\
\hline Superonasal & $31 \%(16-49)$ & $16 \%(06-35)$ & $08 \%(02-19)$ & 0.001 & $<0.001$ & 0.02 \\
\hline Inferonasal & $41 \%(11-63)$ & $18 \%(03-40)$ & $30 \%(18-48)$ & $<0.001$ & 0.06 & 0.04 \\
\hline Inferior & $57 \%(26-76)$ & $34 \%(13-54)$ & $38 \%(23-60)$ & $<0.001$ & 0.002 & 0.52 \\
\hline Inferotemporal & $43 \%(16-77)$ & $31 \%(14-53)$ & $33 \%(18-50)$ & 0.04 & 0.10 & 0.74 \\
\hline Average & $51 \%(33-71)$ & $25 \%(02-40)$ & $16 \%(06-30)$ & $<0.001$ & $<0.001$ & 0.06 \\
\hline
\end{tabular}

Abbreviations: GCIPL, ganglion cell-inner plexiform layer; SAP, standard automated perimetry; MP, microperimetry; $P_{-} 1, P$-value associated with the comparison of the sensitivities between GCIPL and SAP parameters; P_2, P-value associated with the comparison of the sensitivities between GCIPL and MP parameters; P_3, $P$-value associated with the comparison of the sensitivities between SAP and MP parameters. ${ }^{a} 95 \%$ Confidence intervals within parentheses.

a

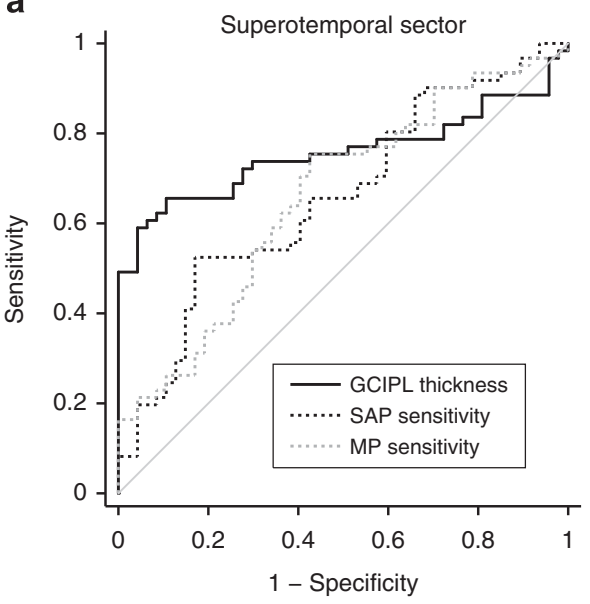

C

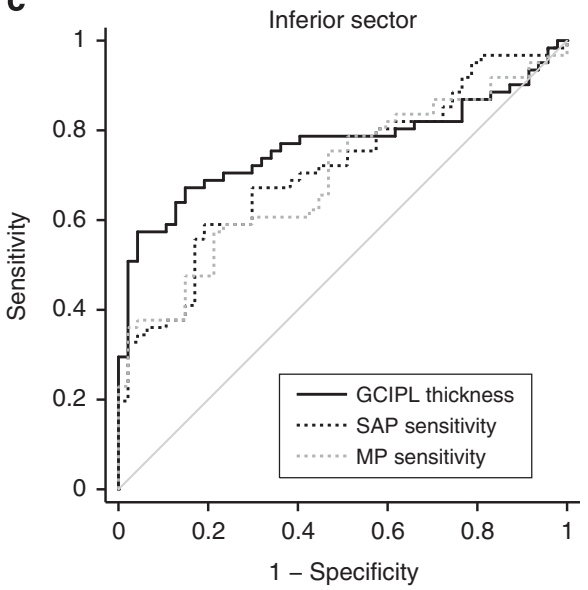

b

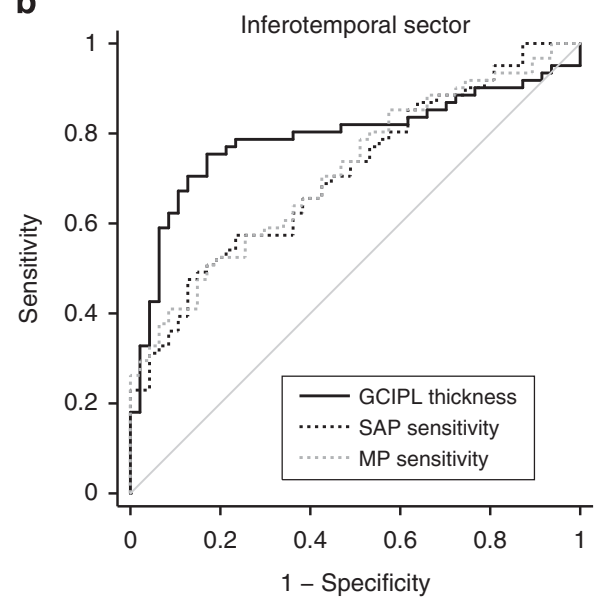

d

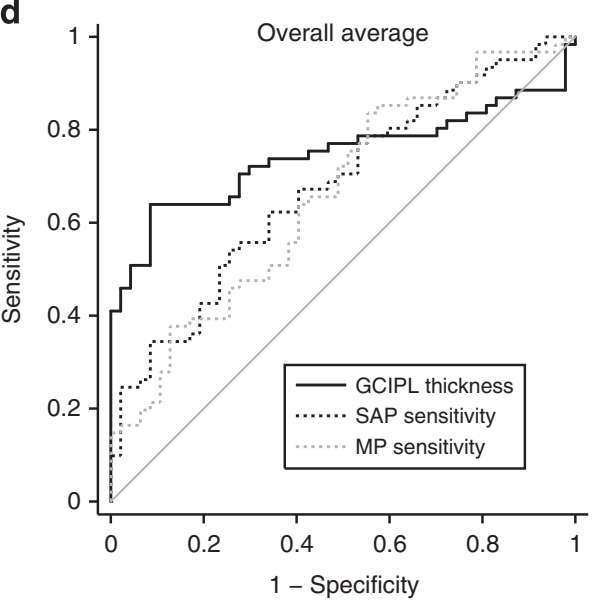

Figure 2 ROC curves showing the diagnostic abilities of ganglion cell-inner plexiform layer (GCIPL) thickness and standard automated perimetry (SAP) and microperimetry (MP) sensitivities of (a) superotemporal sector, (b) inferotemporal sector, (c) inferior sector, and (d) overall average measurement.

(glaucoma defined based on optic disc changes and VF defects on 24-2 SAP). ${ }^{25}$ They found that the AUCs of the mean sensitivities of 10-2 SAP and MP in diagnosing glaucoma were 0.75 and 0.65 , respectively, and that of
CSLO was 0.59 . They concluded that the functional defects occur before anatomic defects in glaucoma. ${ }^{25}$ In another study, Klamann et al ${ }^{26}$ compared the diagnostic abilities of the mean sensitivity of MP with that of the 
RNFL thickness of OCT and CSLO in preperimetric glaucoma (defined as glaucomatous optic neuropathy with normal 24-2 SAP results). They, in contrast to the previous study, found that the AUC of the mean sensitivity with MP was 0.59 , while that of the RNFL thickness with CSLO and OCT were 0.70 and 0.89 , respectively. They concluded that the morphological changes appear earlier than the functional changes in preperimetric glaucoma. ${ }^{26}$

A few earlier studies comparing SAP with MP have also reported better ability of MP to detect VF defects compared with SAP. In 20 glaucomatous eyes with paracentral VF defects (defects involving the central $10^{\circ}$ of VF on a $24^{\circ}$ test), Lima et $a l^{27}$ found that the visual sensitivities of MP in the central $10^{\circ}$ of VF not only correlated significantly with those from 10-2 SAP but also detected abnormal areas of retinal sensitivity in quadrants with normal SAP values. They also found that $75 \%$ of the areas showing abnormal visual sensitivity with MP and normal sensitivity with SAP showed a corresponding reduction in macular thickness on time domain OCT. ${ }^{27}$ In a previous study with the first version of MP, Orzalesi et $a l^{28}$ had also found reduction in visual sensitivities with MP in areas of localized RNFL defects but with normal SAP results. However, we found no consistent difference between the diagnostic abilities of MP and SAP. The AUCs of all SAP and MP visual sensitivity measurements were comparable. Sensitivity of SAP at $95 \%$ specificity was better than that of MP in the superior and superonasal sectors while the same was lesser than that of MP in the inferonasal sector.

An important difference between our study and most of these previous studies is the way cases and controls were defined. It is difficult to define an independent reference standard for glaucoma when comparing structural and functional tests without favoring either one of them. ${ }^{29}$ Previous studies defined glaucoma based on the presence of ONH changes as well as VF defects on SAP. ${ }^{25,27}$ This definition is likely to favor the functional measurements. We defined glaucoma based on the clinical examination of optic disc and RNFL. Our definition was not dependent on the results of SAP or SDOCT. The definition of glaucoma cannot be based on clinical examination of the macula at this point in time. We therefore believed that the definition of glaucoma in our study neither favored the structural nor the functional tests. However, the possibility of our definition favoring the structural test (GCIPL measurement) cannot be ruled out completely as a previous study by Kim et $a l^{30}$ has shown that the diagnostic ability of GCIPL parameters is affected by the topographic profiles of RNFL defects. We however did not evaluate the profile of RNFL defects in our study and therefore cannot rule out this possibility completely. Our study is an initial step in comparing the structural and functional tests at macula in diagnosing glaucoma and should not be extrapolated to the situation of detecting disease in suspects. Future studies should evaluate the structural and functional changes at macula in suspects with high IOP (who would have a higher prior probability of glaucoma) against a normotensive control group.

One of the limitations of the study is the small sample size. The other limitation was the inclusion of two eyes of a few subjects for the analysis. We however accounted for the correlation between two eyes of subjects using validated statistical methods during the analysis. We also ran the analysis with one randomly chosen eye per subject and found the results to be essentially the same. We also did not evaluate the normative database classification provided by SDOCT and its agreement with SAP and MP results in this study. There are two reasons for this. First is that the diagnostic ability of the normative database classification of GCIPL parameters has been reported by previous studies. ${ }^{9,31}$ Second is the fact that there are no validated normative database classification systems with the central $10^{\circ}$ program of either SAP or MP for diagnosing glaucoma. Future studies should also focus on developing and evaluating different classification systems based on the sensitivity or the probability maps of 10-2 SAP and MP for diagnosing glaucoma.

In conclusion, on evaluating the diagnostic abilities of structural and functional tests at macula in glaucoma, GCIPL thickness measurements with HD-OCT performed significantly better than the visual sensitivity measurements by SAP and MP.

\section{Summary}

What was known before

- The diagnostic ability of inner retinal layer measurements at macula with SDOCT in glaucoma.

- The utility of 10-2 SAP program in picking up central VF defects not picked up by 24-2 program.

\section{What this study adds}

- It compares the diagnostic abilities of inner retinal layers at macula with that of the visual fields in picking up glaucoma based on clinical examination of $\mathrm{ONH}$ and RNFL.

\section{Conflict of interest}

The authors declare no conflict of interest.

\section{Acknowledgements}

Rao HL: Allergan (C), Cipla (C); Hussain SM: none; Januwada M: none; Pillutla LN: none; Begum VU: none; 
Chaitanya A: none; Senthil S: none; Garudadri CS: Alcon (C).

\section{References}

1 Rao HL, Zangwill LM, Weinreb RN, Sample PA, Alencar LM, Medeiros FA. Comparison of different spectral domain optical coherence tomography scanning areas for glaucoma diagnosis. Ophthalmology 2010; 117: 1692-1699.

2 Rao HL, Babu JG, Addepalli UK, Senthil S, Garudadri CS. Retinal nerve fiber layer and macular inner retina measurements by spectral domain optical coherence tomograph in Indian eyes with early glaucoma. Eye (Lond) 2012; 26: 133-139.

3 Takayama K, Hangai M, Durbin M, Nakano N, Morooka S, Akagi $\mathrm{T}$ et al. A novel method to detect local ganglion cell loss in early glaucoma using spectral-domain optical coherence tomography. Invest Ophthalmol Vis Sci 2012; 53 : 6904-6913.

4 Mwanza JC, Durbin MK, Budenz DL, Sayyad FE, Chang RT, Neelakantan A et al. Glaucoma diagnostic accuracy of ganglion cell-inner plexiform layer thickness: comparison with nerve fiber layer and optic nerve head. Ophthalmology 2012; 119: 1151-1158.

5 Kotowski J, Folio LS, Wollstein G, Ishikawa H, Ling Y, Bilonick RA et al. Glaucoma discrimination of segmented cirrus spectral domain optical coherence tomography (SD-OCT) macular scans. Br J Ophthalmol 2012; 96: 1420-1425.

6 Jeoung JW, Choi YJ, Park KH, Kim DM. Macular ganglion cell imaging study: glaucoma diagnostic accuracy of spectral-domain optical coherence tomography. Invest Ophthalmol Vis Sci 2013; 54: 4422-4429.

7 Akashi A, Kanamori A, Nakamura M, Fujihara M, Yamada Y, Negi A. Comparative assessment for the ability of Cirrus, RTVue, and 3D-OCT to diagnose glaucoma. Invest Ophthalmol Vis Sci 2013; 54: 4478-4484.

8 Nouri-Mahdavi K, Nowroozizadeh S, Nassiri N, Cirineo N, Knipping S, Giaconi J et al. Macular ganglion cell/inner plexiform layer measurements by spectral domain optical coherence tomography for detection of early glaucoma and comparison to retinal nerve fiber layer measurements. Am J Ophthalmol 2013; 156: 1297-1307.

9 Begum VU, Addepalli UK, Yadav RK, Shankar K, Senthil S, Garudadri CS et al. Ganglion cell-inner plexiform layer thickness of high definition optical coherence tomography in perimetric and preperimetric glaucoma. Invest Ophthalmol Vis Sci 2014; 55: 4768-4775.

10 Drasdo N, Millican CL, Katholi CR, Curcio CA. The length of Henle fibers in the human retina and a model of ganglion receptive field density in the visual field. Vision Res 2007; 47: 2901-2911.

11 Hood DC, Raza AS, de Moraes CG, Liebmann JM, Ritch R. Glaucomatous damage of the macula. Prog Retin Eye Res 2013; 32: 1-21.

12 Schiefer U, Flad M, Stumpp F, Malsam A, Paetzold J, Vonthein $\mathrm{R}$ et al. Increased detection rate of glaucomatous visual field damage with locally condensed grids: a comparison between fundus-oriented perimetry and conventional visual field examination. Arch Ophthalmol 2003; 121: $458-465$.
13 Traynis I, De Moraes CG, Raza AS, Liebmann JM, Ritch R, Hood DC. Prevalence and nature of early glaucomatous defects in the central 10 degrees of the visual field. JAMA Ophthalmol 2014; 132: 291-297.

14 Rohrschneider K, Bultmann S, Springer C. Use of fundus perimetry (microperimetry) to quantify macular sensitivity. Prog Retin Eye Res 2008; 27: 536-548.

15 Mwanza JC, Oakley JD, Budenz DL, Anderson DR. Ability of cirrus HD-OCT optic nerve head parameters to discriminate normal from glaucomatous eyes. Ophthalmology 2011; 118: 241-248.

16 Mwanza JC, Oakley JD, Budenz DL, Chang RT, Knight OJ, Feuer WJ. Macular ganglion cell-inner plexiform layer: automated detection and thickness reproducibility with spectral domain-optical coherence tomography in glaucoma. Invest Ophthalmol Vis Sci 2011; 52: 8323-8329.

17 Sato S, Hirooka K, Baba T, Tenkumo K, Nitta E, Shiraga F. Correlation between the ganglion cell-inner plexiform layer thickness measured with cirrus HD-OCT and macular visual field sensitivity measured with microperimetry. Invest Ophthalmol Vis Sci 2013; 54: 3046-3051.

18 Hood DC, Kardon RH. A framework for comparing structural and functional measures of glaucomatous damage. Prog Retin Eye Res 2007; 26: 688-710.

19 Glynn RJ, Rosner B. Accounting for the correlation between fellow eyes in regression analysis. Arch Ophthalmol 1992; 110: 381-387.

20 Zhou XH, Obuchowski NA, McClish DK. Analysis of correlated ROC data. In: Zhou XH, Obuchowski NA, McClish DK (eds). Statistical Methods in Diagnostic Medicine. Wiley: New York, NY, USA, 2002, pp 274-306.

21 Alonzo TA, Pepe MS. Distribution-free ROC analysis using binary regression techniques. Biostatistics 2002; 3: 421-432.

22 Zhou XH, Obuchowski NA, McClish DK. Comparing the accuracy of two diagnostic tests. In: Zhou XH, Obuchowski NA, McClish DK (eds). Statistical Methods in Diagnostic Medicine. Wiley: New York, NY, USA, 2002, pp 165-194.

23 DeLong ER, DeLong DM, Clarke-Pearson DL. Comparing the areas under two or more correlated receiver operating characteristic curves: a nonparametric approach. Biometrics 1988; 44: 837-845.

24 Pepe MS. The Statistical Evaluation of Medical Tests for Classification and Prediction. Oxford University Press: Oxford, UK, 2003, pp 130-167.

25 Ozturk F, Yavas GF, Kusbeci T, Ermis SS. A comparison among Humphrey field analyzer, microperimetry, and Heidelberg retina tomograph in the evaluation of macula in primary open angle glaucoma. J Glaucoma 2008; 17: 118-121.

26 Klamann MK, Grunert A, Maier AK, Gonnermann J, Joussen AM, Huber KK. Comparison of functional and morphological diagnostics in glaucoma patients and healthy subjects. Ophthalmic Res 2013; 49: 192-198.

27 Lima VC, Prata TS, De Moraes CG, Kim J, Seiple W, Rosen RB et al. A comparison between microperimetry and standard achromatic perimetry of the central visual field in eyes with glaucomatous paracentral visual-field defects. $\mathrm{Br} \mathrm{J}$ Ophthalmol 2010; 94: 64-67.

28 Orzalesi N, Miglior S, Lonati C, Rosetti L. Microperimetry of localized retinal nerve fiber layer defects. Vision Res 1998; 38: 763-771.

29 Garway-Heath DF, Hitchings RA. Sources of bias in studies of optic disc and retinal nerve fibre layer morphology. $\mathrm{Br} J$ Ophthalmol 1998; 82: 986. 
30 Kim MJ, Jeoung JW, Park KH, Choi YJ, Kim DM.

Topographic profiles of retinal nerve fiber layer defects affect the diagnostic performance of macular scans in

preperimetric glaucoma. Invest Ophthalmol Vis Sci 2014; 55: 2079-2087.
31 Mwanza JC, Budenz DL, Godfrey DG, Neelakantan A, Sayyad FE, Chang RT et al. Diagnostic performance of optical coherence tomography ganglion cell-inner plexiform layer thickness measurements in early glaucoma. Ophthalmology 2014; 121: 849-854. 